\title{
KINEMATICS IN A COMPLEX PLANE AND SOME GEOMETRIC APPLICATIONS *
}

\author{
BY ARNOLD EMCH
}

1. Introduction. From an elementary standpoint one is apt to consider geometry in a complex plane as an accessory of heuristic value for function theory. In a more advanced sense one recognizes the fundamental importance and intrinsic value of the geometric problem of partition of the complex plane by circular arcs in connection with the properties and classification of certain linear substitution groups, with the corresponding automorphic functions, and, in particular, with the theory of algebraic curves and their Riemann surfaces and uniformization. But even in a more elementary sense, the complex plane is the natural medium for the solution of certain specific geometric problems. As an example may be mentioned the "geometry of the polynomial," involving the theory of stelloid and circular curves and their focal properties. $\dagger$

Also a number of problems in geometric kinematics may be solved conveniently in a complex plane, as has been shown by Koenigs, $\ddagger$ Study, $\S$ and others. In the present paper I shall show by further examples of this kind the simplicity and elegance of the complex treatment.

2. Similar Triangles. As can easily be verified, a necessary and sufficient condition for the equi-sensed similitude of two triangles $z_{1} z_{2} z_{3}, z_{1}{ }^{\prime} z_{2}{ }^{\prime} z_{3}{ }^{\prime}$ is the vanishing of the determinant

$$
\left|\begin{array}{lll}
z_{1} & z_{1}^{\prime} & 1 \\
z_{2} & z_{2}^{\prime} & 1 \\
z_{3} & z_{3}^{\prime} & 1
\end{array}\right| \equiv 0 .
$$

* Presented to the Society, Feb. 25, 1922.

$\dagger$ See Emch, On a certain generation of rational circular and isotropic curves, this Bulletin, vol. 25, pp. 397-404 (1919), and also On plane algebraic curves with a given system of foci, same volume, pp. 157-161.

$\ddagger$ Leçons de Cinématique: Les imaginaires dans la cinématique du plan, pp. 324-332 (1897).

$\S$ Vorlesungen über ausgewählte Gegenstände der Geometrie. Erstes Heft: Ebene analytische Kurven und zu ihnen gehörige Abbildungen, pp. 1-18 (1911). 
Denoting by $\bar{z}$ the conjugate of $z$, two triangles $z_{1} z_{2} z_{3}, z_{1}{ }^{\prime} z_{2}{ }^{\prime} z_{3}{ }^{\prime}$ are similar with the sense inverted when

$$
\left|\begin{array}{lll}
\bar{z}_{1} & z_{1}^{\prime} & 1 \\
\bar{z}_{2} & z_{2}^{\prime} & 1 \\
\bar{z}_{3} & z_{3}{ }^{\prime} & 1
\end{array}\right| \equiv 0 .
$$

For an equilateral triangle $z_{1} z_{2} z_{3}$ we simply have to set up the condition that the triangle $z_{2} z_{3} z_{1}$ in the same order be similar to the triangle $z_{1} z_{2} z_{3}$; i.e.,

This reduces to

$$
\left|\begin{array}{lll}
z_{1} & z_{2} & 1 \\
z_{2} & z_{3} & 1 \\
z_{3} & z_{1} & 1
\end{array}\right| \equiv 0 .
$$

$$
z_{1}^{2}+z_{2}^{2}+z_{3}^{2}-z_{2} z_{3}-z_{3} z_{1}-z_{1} z_{2}=0
$$

as a necessary and sufficient condition that the triangle $z_{1} z_{2} z_{3}$ be equilateral.

3. The Group of Movements in a Plane. The linear transformation

$$
z^{\prime}=a z+b
$$

may be considered as a movement (including uniform dilatation) of the $z$-plane into a new position indicated by $z^{\prime}$ and referred to the same system of coordinates (original z-plane). Putting $a=r_{1} e^{i a_{1}}, b=r_{2} e^{i a_{2}}, z=r e^{i \theta}$, (4) becomes

$$
z^{\prime}=r_{1} r e^{i\left(\theta+a_{1}\right)}+r_{2} e^{i a_{2}},
$$

and it is seen at once that the movement is equivalent to the effect of the succession of substitutions
(a) $S_{1}$ (similitude): $\quad z_{1}=r_{1} \cdot z$;
(b) $S_{2}\left(\right.$ rotation): $\quad z_{2}=z_{1} \cdot e^{i a_{1}}$;
(c) $S_{3}$ (translation): $\quad z_{3}=z^{\prime}=z_{2}+r_{2} e^{i a_{2}}$.

The totality of all movements (4) forms a continuous projective four-parameter group and contains $(a),(b),(c)$ as subgroups. The invariant points in the movement from the $z-$ to the $z^{\prime}$-plane are $z=b /(1-a)$ and $z=\infty$.

When $z$ describes a figure in the original plane, $z^{\prime}$ describes a similar figure in the displaced plane with the coefficient of dilatation equal to $r_{1}$. In particular, when $z$ describes either a straight line or a circle, $z^{\prime}$ will describe a straight line or a 
circle. Moreover, since

$$
d z^{\prime}=a \cdot d z, \quad \text { and } \quad\left|d z^{\prime}\right|=r_{1} \cdot|d z|,
$$

the ratio of the velocities of $z$ and $z^{\prime}$ is constant. Thus when $z$ describes a straight line with uniform velocity, $z^{\prime}$ will describe a straight line with uniform velocity. When $z$ describes a circle, $z^{\prime}$ describes a circle with the same angular velocity, i.e., $z$ and $z^{\prime}$ describe their respective circles in the same time.

\section{On the Movement of a Triangle in Deformation and Remain-} ing Similar to Itself. When $z$ describes the unit-circle $z=e^{i \theta}$,

$$
z^{\prime}=r_{1} e^{i\left(\theta+a_{1}\right)}+r_{2} e^{i a_{2}}
$$

describes obviously a circle with $r_{2} e^{i a_{2}}$ as a center and $r_{1}$ as a radius. Consider now the triangle formed by the origin, the unit-point on the real axis and the fixed point $\rho e^{i \beta}$. On the line $z z^{\prime}$ which connects the point $z$ on the unit-circle to the point $z^{\prime}$, corresponding to $z$ by (4), erect a triangle $z_{3} z z^{\prime}$ similar, in the same order, to the triangle $\rho e^{i \beta}, 0,1$. Then

$$
\left|\begin{array}{lll}
z_{3} & \rho e^{i \beta} & 1 \\
e^{i \theta} & 0 & 1 \\
r_{1} e^{i\left(\theta+a_{1}\right)}+r_{2} e^{i a_{2}} & 1 & 1
\end{array}\right|=0 .
$$

From this

$$
\begin{gathered}
-z_{3}+e^{i \theta}-\rho e^{i(\theta+\beta)}+\rho r_{1} e^{i\left(\theta+\alpha_{1}+\beta\right)}+\rho r_{2} e^{i\left(\alpha_{2}+\beta\right)}=0 \\
z_{3}=\left\{\rho r_{1} e^{i\left(\alpha_{1}+\beta\right)}-\rho e^{i \beta}+1\right\} e^{i \theta}+\rho r_{2} e^{i\left(\alpha_{2}+\beta\right)}
\end{gathered}
$$

As (7) has the same form as (6) and $\theta$ is the only variable, $z_{3}$ clearly describes a circle with $\left|\rho r_{1} e^{i\left(a_{1}+\beta\right)}-\rho e^{i \beta}+1\right|$ as a radius and $\rho r_{2} e^{i\left(a_{2}+\beta\right)}$ as a center. Obviously, $z_{3}, z^{\prime}, z$ describe the circles with the same angular velocities, i.e., they simultaneously describe the circles completely. Denote the centers of the circles described by $z, z^{\prime}, z_{3}$ by $c_{1}, c_{2}, c_{3}$, so that $c_{1}=0$, $c_{2}=r_{2} e^{i a_{8}}, c_{3}=\rho r_{2} e^{i\left(a_{2}+\beta\right)}$. Comparing this triangle with the original fixed triangle $0,1, \rho e^{i \beta}$, we see that

$$
\left|\begin{array}{lll}
0 & 0 & 1 \\
r_{2} e^{i a_{2}} & 1 & 1 \\
\rho r_{2} e^{i\left(a_{2}+\beta\right)} & \rho e^{i \beta} & 1
\end{array}\right| \equiv 0 .
$$

From this it follows that the two triangles are similar.

Consider next a point $z_{4}$ which remains always similarly attached to the triangle $z z^{\prime} z_{3}$, so that also $z z^{\prime} z_{4}$ remains similar 
to itself. But such a triangle $z z^{\prime} z_{4}$ arises from a definite fixed similar triangle $0,1, \delta e^{i \nu}$, just as $z z^{\prime} z_{3}$ arises from $0,1, \rho e^{i \beta}$. Hence also $z_{4}$ describes a circle, and $\triangle c_{1} c_{2} c_{4} \sim \triangle z z^{\prime} z_{4}$, i.e., if a fourth point $z_{4}$ remains similarly attached to the triangle $z z_{1} z_{3}$, then $z_{4}$ describes a circle whose center $c_{4}$ is similarly attached to $c_{1} c_{2} c_{3}$. The same situation prevails for other similarly attached points $z_{5}, z_{6}, \cdots$, and as the geometric result does not depend upon the orientation of the complex plane, we may state the following theorem.

Theorem 1. If a variable closed or unclosed polygon

$$
(z)=z_{0} z_{1} z_{2} \cdots z_{n}
$$

of $(n+1)$ vertices remains similar to some arbitrary fixed polygon

$$
(a)=a_{0} a_{1} a_{2} \cdots a_{n}
$$

and any two of its vertices describe two fixed circles with the same angular velocities, then all other vertices describe circles with the same angular velocities. The polygon

$$
(c)=c_{0} c_{1} c_{2} c_{3} \cdots c_{n}
$$

of the centers of these circles is similar to the fixed polygon (a).

From this follows obviously the corollary:

COROLlary.* If any two vertices of a regular variable polygon $(z)=z_{1} z_{2} \cdots z_{n}$ of $n$ vertices and center $z_{0}$ describe two circles with the same angular velocities, then the remaining $n-2$ vertices describe circles with the same angular velocities. The centers $c_{1} c_{2} c_{3} \cdots c_{n}$ of these circles form a fixed regular polygon $(c)$, whose center $c_{0}$ is the center of the circle described by the center $z_{0}$ of the variable polygon (z).

5. Case of Movements on Straight Lines. It is not difficult to show that the theorem of the preceding section holds, when two points of the polygon $(z)$ describe straight lines with uniform velocities. We may restate the result.

Theorem 2. If any two points of a variable polygon (z), which remains similar to some fixed polygon (a), describe two straight lines with uniform velocities, then all other points of (z) describe straight lines with uniform velocities.

* This contains Study's theorem on the square, loc. cit., p. 16, as a particular case, It is obviously not necessary to make a restriction in the choice of the two vertices as Study does. 
In particular, when (z) is a regular polygon, its center $z_{0}$ also describes a straight line.

We propose next to discover whether it is possible to find triangles with their vertices on three arbitrarily given fixed lines and similar to a fixed triangle $0,1, r e^{i a}$. Let $\lambda_{1}, \lambda_{2}, \lambda_{3}$ be three variable parameters; then the three lines may be written in the form

$$
\begin{aligned}
& z_{1}=a_{1}+\lambda_{1} e^{i a_{1}}, \\
& z_{2}=a_{2}+\lambda_{2} e^{i a_{2}}, \\
& z_{3}=a_{3}+\lambda_{3} e^{i a_{3}} .
\end{aligned}
$$

For every set of values $\lambda_{1}, \lambda_{2}, \lambda_{3}$ the three points $z_{1}, z_{2}, z_{3}$ form a triangle on the three lines. Similitude to the fixed triangle requires

$$
\left|\begin{array}{lll}
a_{1}+\lambda_{1} e^{i a_{1}} & 0 & 1 \\
a_{2}+\lambda_{2} e^{i_{2}} & 1 & 1 \\
a_{3}+\lambda_{3} e^{i a_{3}} & r e^{i a} & 1
\end{array}\right|=0 .
$$

Expanding this and separating real from imaginary, we get an expression of the type

$$
A_{1} \lambda_{1}+A_{2} \lambda_{2}+A_{3} \lambda_{3}+A_{4}+i\left(B_{1} \lambda_{1}+B_{2} \lambda_{2}+B_{3} \lambda_{3}+B_{4}\right)=0
$$

in which the $A$ 's and $B$ 's and $\lambda$ 's are real. In order that this be satisfied for real values of the $\lambda$ 's, there must be

$$
\begin{aligned}
& A_{1} \lambda_{1}+A_{2} \lambda_{2}+A_{3} \lambda_{3}+A_{4}=0, \\
& B_{1} \lambda_{1}+B_{2} \lambda_{2}+B_{3} \lambda_{3}+B_{4}=0,
\end{aligned}
$$

which shows the existence of a simply infinite set of solutions $\left(\lambda_{1}, \lambda_{2}, \lambda_{3}\right)$, or of such triangles. Hence we have the following theorem.

Theorem 3. If three lines $l_{1}, l_{2}, l_{3}$ are given, it is always possible to describe them simultaneously by three points of a oneparameter variable triangle which remains similar to a fixed triangle. The vertices of the triangle describe the sides $l_{1} l_{2} l_{3}$ with definite uniform velocities.

Now choose a fourth point $z_{4}$ so that the variable quadrangle $z_{1} z_{2} z_{3} z_{4}$, with $z_{1}, z_{2}, z_{3}$ moving on $l_{1} l_{2} l_{3}$, remains similar to a fixed quadrangle $a_{1} a_{2} a_{3} a_{4}$; then $z_{4}$ describes a straight line $l_{4}$. 
If four lines $l_{0} l_{1} l_{2} l_{3}$ are given, and we let $z_{1}, z_{2}, z_{3}$ describe $l_{1}, l_{2}, l_{3}$ as before, then $z_{4}$ describes a line $l_{4}$. When $z_{4}$, moving on $l_{4}$, reaches the intersection $z_{0}^{\prime}$ of $l_{4}$ with $l_{0}, z_{1}, z_{2}, z_{3}$ will have reached certain positions $z_{1}{ }^{\prime} z_{2}{ }^{\prime} z_{3}{ }^{\prime}$ on $l_{1}, l_{2}, l_{3}$, respectively, so that $z_{0}{ }^{\prime} z_{1}{ }^{\prime} z_{2}^{\prime} z_{3}{ }^{\prime} \sim a_{1} a_{2} a_{3} a_{4}$.

Theorem 4. It is always possible to inscribe in a given quadrilateral a quadrangle similar to a given quadrangle. In particular, it is always possible to inscribe a square in a given quadrangle.*

Theorem I is also true when one of the two circles determining the movement degenerates into a point circle, or when one degenerates into a point circle and the other into a straight line. Thus follows

Theorem 5 . If a variable triangle $z_{1} z_{2} z_{3}$ remains similar to a fixed triangle, and $z_{3}$ is stationary, while $z_{1}$ describes a straight line with uniform velocity, then $z_{2}$ describes another straight line with uniform velocity.

As a particular case assume an isosceles right triangle $z_{1} z_{2} z_{3}$ with $z_{3}=e i$ fixed on the axis of imaginaries and $z_{1}=\lambda$, the vertex of the right angle, describing the axis of reals. Then $z_{2}-\lambda=-(e i-\lambda) i$, and $z_{2}=e+\lambda(1+i)$, i.e., $z_{2}$ describes a line through the point $e$ on the real axis and making an angle of $45^{\circ}$ with the positive part of the real axis. This result leads to the following method.

6. Inscribing a Square in a Quadrilateral. Let $l_{1} l_{2} l_{3} l_{4}$ be the sides of a proper quadrilateral following each other in the positive sense. On $l_{1}$ choose a trial point $A_{1}$ and drop a perpendicular $A_{1} Q_{1}$ on $l_{2}$. From $Q_{1}$ measure off on $l_{2}$ in the positive sense $Q_{1} A_{2}{ }^{\prime}=A_{1} Q_{1}$. Through $A_{2}{ }^{\prime}$ draw a line $g_{1}$ making an angle of $45^{\circ}$ with the positive direction of $l_{2}$, and intersecting $l_{3}$ in $A_{3}$. Then $A_{1} A_{3}$ form two opposite vertices of a square on $l_{1} l_{2} l_{3}$ and we can easily find the other vertices $A_{2}, A_{4}$. Repeat the same construction, starting with a second

* The second part of this theorem is well known. See Dr. Hebbert's papers, Annals of Mathematics (2), vol. 16, pp. 38-42; 61-71 (191415), and references given there. 
trial point $B_{1}$ on $l_{1}$, resulting in a second square $B_{1} B_{2} B_{3} B_{4}$ with $B_{1} B_{2} B_{3}$ on $l_{1} l_{2} l_{3}$. Now when a variable square moves with three vertices on three lines $l_{1} l_{2} l_{3}$, the fourth vertex describes a line $l_{4}{ }^{\prime} . \quad A_{1} A_{2} A_{3} A_{4}$ and $B_{1} B_{2} B_{3} B_{4}$ are two positions of this square, and $A_{4}$ and $B_{4}$ determine the line $l_{4}{ }^{\prime}$. Hence, the point of intersection $P_{4}$ of $l_{4}{ }^{\prime}$ and $l_{4}$ is a vertex of the inscribed square. Repeating the above construction in the reversed order, starting with $P_{4}$ on $l_{4}$, easily gives the square $P_{4} P_{3} P_{2} P_{1}$ inscribed in $l_{1} l_{2} l_{3} l_{4}$.

This however is not the only square. A second square is obtained by measuring off $Q_{1} A_{2}{ }^{*}=-Q_{1} A_{2}$ in the negative sense, by drawing $g_{1}{ }^{*}$ through $A_{2}{ }^{*}$, making an angle of $45^{\circ}$ with the negative direction of $l_{2}$, and proceeding with the construction as explained above. Starting again with $l_{1}$ and choosing the order $l_{1} l_{2} l_{4} l_{3}$, there are again two squares whose vertices lie in succession on $l_{1} l_{2} l_{4} l_{3}$. Likewise there are two squares for the order $l_{1} l_{3} l_{2} l_{4}$. The remaining three orders $l_{1} l_{4} l_{3} l_{2}, l_{1} l_{3} l_{4} l_{2}, l_{1} l_{4} l_{2} l_{3}$ are cyclic substitutions of the above three and do not produce any new squares.

THEOREM 6. There are, in general, six squares that may be inscribed in a proper quadrilateral. For each of the three noncyclic orders there are two squares of opposite sense.

When there exist two inscribed squares with the same sense for a given order, as for $l_{1} l_{2} l_{3} l_{4}$, then $l_{4}{ }^{\prime}$ coincides with $l_{4}$, and there exists an infinite number of solutions.

Theorem 7. When for a given order of a quadrilateral there exist two inscribed squares with the same sense, then there exist an infinite number of inscribed squares for the same order.

Thus a problem of old standing is solved in a complete* and very simple manner by preparing the way in the complex field.

UNIVERSITY OF ILLINOIS

* Carnot in his trigonometric solution of the problem (Géométrie de Position, p. 374) stated the possibility of three solutions only. 